\title{
éditionsOCDE
}

\section{LES NOUVELLES OPPORTUNITÉS DE L'ÉCONOMIE ALIMENTAIRE OUEST-AFRICAINE}

\section{NOTES OUEST-AFRICAINES}

Juillet $2016 \mathbf{N}^{\circ} 01$

Secrétariat du

Clubs) 



\section{NOTES OUEST-AFRICAINES}

\section{LES NOUVELLES OPPORTUNITÉS \\ DE L'ÉCONOMIE ALIMENTAIRE OUEST-AFRICAINE}

Cette note a été rédigée par

THOMAS ALLEN

PHILIPP HEINRIGS

Secrétariat du Club du Sahel et de l'Afrique de l'Ouest/OCDE 


\section{NOTES OUEST-AFRICAINES}

La série Notes ouest-africaines vise à partager des études avec une large audience d'experts, de praticiens du développement, de décisionnaires et de lecteurs avertis. Cette série analyse la nature, les dynamiques et les caractéristiques des transformations socio-économiques et politiques que traverse l'Afrique dans une perspective régionale et multidisciplinaire. Elle cherche à stimuler la discussion, rassembler les informations et mieux anticiper les transformations en cours pour les politiques à venir. Les Notes sont disponibles en anglais et/ou en français ; les résumés dans les deux langues. Initiées par le Club du Sahel et de l'Afrique de l'Ouest (CSAO) pour éclairer les enjeux ouest-africains, ces analyses sont préparées par son Secrétariat, ses membres et partenaires, les autres départements de l'OCDE, des organisations internationales et autres experts.

En savoir plus sur le Club du Sahel et de l'Afrique de l'Ouest : http://www.oecd.org/fr/csao.

Merci de citer cet ouvrage comme suit :

Allen, T. et P. Heinrigs (2016), ( Les nouvelles opportunités de l'économie alimentaire ouestafricaine »), Notes ouest-africaines, $\mathrm{N}^{\circ} 01$, Éditions OCDE, Paris. http://dx.doi.org/10.1787/5jlwjg67125f-fr.

Contact auteurs : thomas.allen@oecd.org et philipp.heinrigs@oecd.org

ISSN 2415-1149

Les documents de travail de l'OCDE ne doivent pas être présentés comme exprimant les vues officielles de l'OCDE ou de ses pays membres. Les opinions exprimées et les arguments employés sont ceux des auteurs.

Ce document et toute carte qu'il peut comprendre ne préjugent en rien du statut de tout territoire, de la souveraineté s'exerçant sur ce dernier, du tracé des frontières et limites internationales, et du nom de tout territoire, ville ou région.

Les documents de travail exposent des résultats préliminaires ou des travaux de recherche en cours menés par l'auteur/les auteurs et sont publiés pour stimuler le débat sur un large éventail de questions sur lesquelles l'OCDE travaille. Les commentaires sur les documents de travail sont bienvenus et peuvent être adressés au Club du Sahel et de l'Afrique de l'Ouest, OCDE, 2 rue André-Pascal, 75775 Paris Cedex 16, France.

Autorisé pour publication par Laurent Bossard, Directeur, Secrétariat du Club du Sahel et de l'Afrique de l'Ouest (CSAO/OCDE).

Vous êtes autorisés à copier, télécharger ou imprimer du contenu OCDE pour votre utilisation personnelle. Vous pouvez inclure des extraits des publications, des bases de données et produits multimédia de l'OCDE dans vos documents, présentations, blogs, sites Internet et matériel d'enseignement, sous réserve de faire mention de la source OCDE et du copyright. Les demandes pour usage commercial ou de traduction devront être adressées à rights@oecd.org. 


\section{RÉSUMÉ}

L'économie alimentaire ouest-africaine s'est profondément transformée ces 60 dernières années sous l'effet de l'urbanisation et de la croissance des revenus. L'ensemble des activités de production, transformation et distribution qui concourent à l'alimentation humaine, représente un total de 178 milliards USD en 2010, soit 36 \% du PIB régional. Environ $40 \%$ de la valeur ajoutée de cette économie alimentaire n'est plus le fait de l'agriculture. En particulier, les segments aval de l'agriculture dans les chaînes de valeur alimentaires gagnent en importance et devraient connaitre la croissance la plus forte sur les prochaines décennies. La région doit se doter des mécanismes de suivi et d'analyse adaptés et revoir ses politiques si elle souhaite tirer profit des nouvelles opportunités en matière de développement agricole, d'emploi et de création de valeur ajoutée. Ce document propose une estimation de la taille et de la structure de cette nouvelle économie alimentaire, et revient sur les principaux enjeux de politique publique qui en découlent.

Mots clés : systèmes alimentaires ; politique alimentaire ; transformation agricole ; aliments transformés ; urbanisation ; Afrique de l'Ouest ; chaînes alimentaires

Code JEL : Q18 ; Q13

\section{TABLE DES MATIÈRES}

INTRODUCTION 4

1 L'IMPORTANCE DES MARCHÉS ALIMENTAIRES ..................................................

1.1 Une urbanisation moteur de changements ........................................................ 5

1.2 De nouvelles habitudes de consommation ........................................................... 5

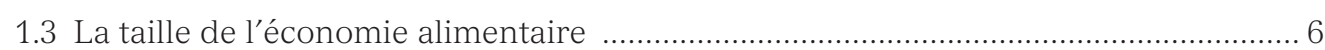

2 UNE ÉCONOMIE ALIMENTAIRE DE MOINS EN MOINS AGRICOLE ............................9

2.1 Création de valeur ajoutée au-delà de l'agriculture ................................................ 9

2.2 Le développement des chaînes de valeur alimentaires .......................................... 12

3 IMPLICATIONS POUR LES POLITIQUES ET LA SÉCURITÉ ALIMENTAIRE .................. 14

3.1 Connecter et coordonner les activités de l'économie alimentaire ............................ 14

3.2 Adapter les outils de la sécurité alimentaire et nutritionnelle.................................. 17

CONCLUSION .................................................................................................................. 19

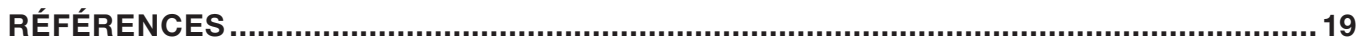




\section{INTRODUCTION}

a forte croissance démographique des 60 dernières années et la dynamique — d'urbanisation qui l'accompagne, ont profondément transformé l'économie alimentaire ouest-africaine. Alors que la région était essentiellement rurale au moment des indépendances, avec une production agricole partagée entre agriculture de subsistance et agriculture d'exportation de produits de rente, $45 \%$ de la population vit aujourd'hui en ville où les échanges alimentaires marchands dominent. À partir des dernières données d'enquêtes de consommation et de dépenses disponibles, ce document propose une estimation de la taille et de la structure de cette nouvelle économie alimentaire, et revient sur les principaux enjeux de politique publique qui en découlent.

- L'économie alimentaire ouest-africaine, regroupant l'ensemble des activités de production, transformation et distribution qui concourent à l'alimentation humaine, représente un total de 178 milliards USD (dollars US) en 2010, soit 36 \% du PIB régional. C'est le premier secteur économique de la région.

- L'urbanisation et les changements de style de vie qui lui sont associés, contribuent à transformer les habitudes de consommation alimentaire. Les ménages consomment plus de fruits et légumes, et plus de viandes et poissons, alors que la part des céréales et légumineuses dans l'alimentation diminue. Les produits transformés représentent par ailleurs 39 \% des dépenses alimentaires des ménages et font leur entrée dans le panier des ménages assez tôt dans l'échelle des revenus.

- Les activités non agricoles, comme la transformation alimentaire, la logistique ou le commerce de détail, gagnent en importance et représentent désormais $40 \%$ de la valeur ajoutée du secteur.

- Les segments aval de l'agriculture dans les chaînes de valeur alimentaires participent de manière plus générale du processus de diversification de l'économie ouestafricaine. Les activités de transformation alimentaire, en particulier, contribuent significativement à la création de valeur ajoutée des industries manufacturières et sont en progression forte.

Ces mutations de l'économie alimentaire sont source de nouveaux enjeux de politique publique. Tout effort visant à améliorer la production agricole se révélera insuffisant si les défis dans les autres segments des chaînes de valeur ne sont pas abordés simultanément. La disponibilité et l'accessibilité physique aux denrées alimentaires sont désormais

\section{L'économie alimentaire ouest- africaine représente un total de 178 milliards USD en 2010, soit $36 \%$ du PIB régional.} conditionnées à la réalisation d'une succession d'activités le long des chaînes de valeur. Une frange de plus en plus importante de la population urbaine et rurale ouest-africaine est dépendante de cette économie alimentaire plus complexe. La sécurité alimentaire et nutritionnelle devient en retour également plus complexe et le résultat d'un plus grand nombre de facteurs. L'appareil décisionnel doit se doter des mécanismes de suivi et d'analyse adaptés. 


\section{L'IMPORTANCE DES MARCHÉS ALIMENTAIRES}

La demande alimentaire, en volume, a été multipliée par cinq en Afrique de l'Ouest depuis 1960. Elle représente en 2010178 milliards USD, dont plus de deux tiers ont fait l'objet d'échanges sur des marchés. Une part importante - et croissante - de cette demande porte sur des produits à plus forte valeur ajoutée, notamment des produits transformés. Ces mutations rapides et profondes s'opèrent au rythme de l'urbanisation.

\subsection{Une urbanisation moteur de changements}

Entre 1950 et 2010, la population de l'Afrique de l'Ouest est passée de 73 à 317 millions d'habitants (OCDE, 2015). Cette croissance s'est accompagnée d'une redistribution spatiale de la population. La population urbaine a été multipliée par 25 en 60 ans, de 5 à 133 millions d'habitants. Alors qu'en 1950, aucun pays n'a un niveau d'urbanisation supérieur à 20 \%, il atteint 42 \% en 2010 au niveau régional. Six pays, dont le Nigéria, affichent un taux égal ou supérieur à 45 \% en 2010. Ces dynamiques de peuplement façonnent les trajectoires économiques, sociales et politiques de la région.

Les villes grossissent en taille et en nombre. La croissance urbaine se manifeste par la concentration dans les plus grandes villes, mais également par le développement d'un réseau de villes moyennes et petites. Alors qu'en 1950, la région comptait 152 agglomérations de plus de 10000 habitants, elle en enregistre en 2010 1947, dont 22 de plus de 1 million d'habitants. La distance moyenne séparant les agglomérations urbaines est divisée par 4, passant de $111 \mathrm{~km}$ à $27 \mathrm{~km}$. Ces villes, reliées par des réseaux de transport et de communication, constituent les nœuds de l'organisation spatiale des échanges et des marchés. Les marchés sont en effet devenus la principale source d’approvisionnement des ménages en denrées alimentaires. La croissance urbaine continue participe de l'intégration de l'espace rural à cette économie de marché et est ainsi l'un des principaux moteurs de la transformation des systèmes de production agro-alimentaires.

Le développement des marchés alimentaires peut se mesurer à l'évolution de la population non agricole. Avec l'urbanisation, une proportion croissante des ménages sort automatiquement des activités agricoles en milieu urbain, mais également en milieu rural où d'autres services et activités se développent. Alors que $50 \%$ de la population ouest-africaine n'est plus engagée dans des activités agricoles, les modes d'acquisition des aliments changent. En milieu urbain, les ménages s'approvisionnent essentiellement sur les marchés, avec en moyenne plus de $90 \%$ de leur panier provenant d'achats réalisés dans les différents circuits de distribution (OCDE, 2013). En milieu rural, la part des approvisionnements marchands représenterait en 2010 presque la moitié de la valeur économique de la consommation alimentaire. Il est estimé qu'au moins deux tiers des besoins alimentaires sont désormais assurés par les marchés au niveau régional.

\subsection{De nouvelles habitudes de consommation}

La composition du panier alimentaire des ménages ouest-africains se transforme à mesure que les revenus augmentent et que les habitudes de consommation évoluent en milieu urbain. Certaines évolutions de la structure de consommation avec les conditions de vie sont bien connues, du point de vue théorique, et considérées comme des lois économiques stables, aussi bien dans l'espace que dans le temps.

Tout d'abord, conformément à la première loi d'Engel, la part du revenu allouée aux dépenses alimentaires diminue de manière significative avec le revenu, passant de 59 \% pour les plus pauvres à $19 \%$ pour les ménages les plus aisés ${ }^{1}$. Compte tenu de la taille de la population la plus défavorisée, le marché alimentaire reste le premier marché d’Afrique de l'Ouest. Le niveau moyen de revenu étant plus élevé en ville, les ménages urbains consacrent $46 \%$ de leurs dépenses totales à l'alimentation contre 60 \% en milieu rural.

1 En excluant la restauration collective et hors domicile. 
Deuxièmement, la composition du panier alimentaire des ménages (Graphique 1.1) révèle des différences importantes par milieu ; les ruraux consomment proportionnellement plus de céréales (et produits à base de céréales) que les urbains qui leur préfèrent les fruits et légumes, les produits carnés et poissons. Cette évolution est conforme à la loi de Bennet qui pose que la proportion de féculents diminue lorsque le revenu augmente. Les ménages procèdent ainsi à des substitutions entre aliments, avec l'abandon d'aliments considérés moins nobles ou inférieurs pour des calories plus chères. La diversité du régime alimentaire s'améliore et des aliments à plus haute teneur en protéines, comme par exemple la viande apparaissent.

La tendance à la baisse de la consommation de céréales et, en particulier, de racines et tubercules tropicales n'implique cependant pas que leur importance diminue dans l'agriculture ouest-africaine. Il s'agit plus d'une transition vers de nouvelles utilisations. Moins consommées directement, ces cultures sont converties en aliments à plus haute valeur ajoutée comme la viande ou les produits transformés (comme l'attiéké, le gari ou les pâtes alimentaires).

Les produits transformés représentent en moyenne 39 \% des consommations alimentaires des ménages, hors céréales et produits amylacés transformés (Tableau 1.1). Les ménages urbains en consomment davantage (41 \%) que les ruraux (36 \%). Il en va de même pour les ménages les plus aisés qui affichent les taux de consommation de produits transformés les plus élevés (49 \%). Ces derniers font cependant déjà partie, et dans des proportions non négligeables, du panier des ménages les moins favorisés (36 \%), aussi bien en milieu urbain qu'en milieu rural. La quantité de services et de valeur ajoutée, incluse dans les aliments, augmente donc au fur et à mesure que le revenu augmente, mais les produits transformés font leur entrée dans le panier des ménages assez tôt dans l'échelle des revenus. Cette tendance est confirmée par d'autres études en Afrique de l'Ouest (Hollinger et Staatz, 2015) et observée en Afrique australe et de l'Est (Tschirley et al., 2015).

\section{Graphique 1.1}

Répartition des consommations alimentaires des ménages par milieu - Afrique de l'Ouest (2010)

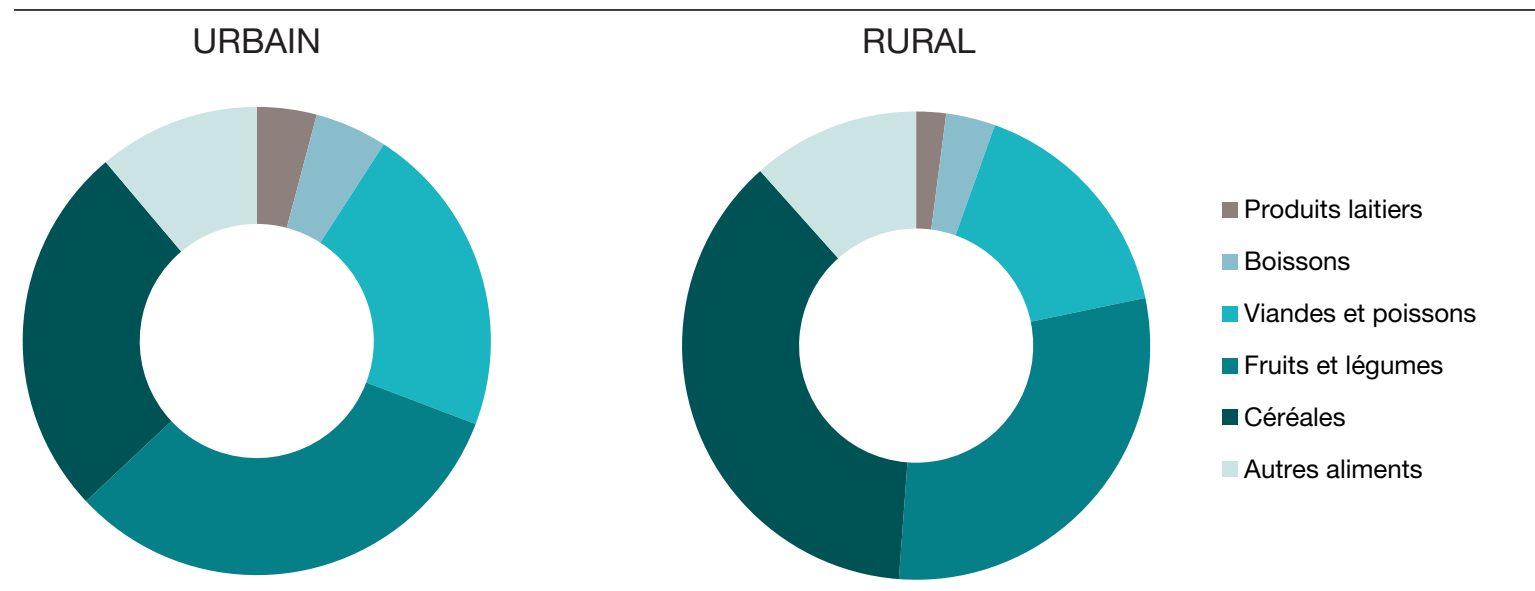

Source : Banque mondiale, 2015 et calculs des auteurs

\subsection{La taille de l'économie alimentaire}

Alimenté par la croissance de la population, l'urbanisation et la hausse des revenus, le secteur alimentaire ouest-africain est plus important que généralement envisagé et en croissance rapide. Les données des comptes nationaux sont insuffisantes pour mesurer l'importance de ce secteur. Une estimation plus fine de sa taille est pour la première fois possible à partir des

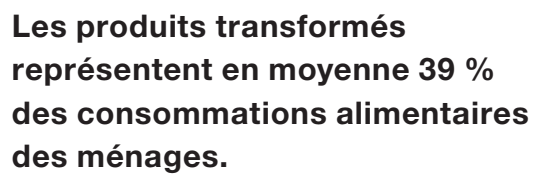

Les produits transformés représentent en moyenne $39 \%$ des consommations alimentaires des ménages. enquêtes de consommation et de dépenses récemment compilées et agrégées par la Banque mondiale. En isolant les consommations de denrées alimentaires des ménages, il est possible de calculer l'économie alimentaire comme 
Tableau 1.1

Parts des aliments transformés et non transformés dans les consommations alimentaires des ménages par milieu et classe de revenu - Afrique de l'Ouest (2010)

\begin{tabular}{|c|c|c|c|c|c|}
\hline & \multirow{2}{*}{ Classe de revenu* } & \multirow{2}{*}{ Boissons } & \multirow{2}{*}{ Céréales** } & \multicolumn{2}{|c|}{ Aliments (hors céréales \& boissons) } \\
\hline & & & & Non transformés & Transformés ${ }^{* * *}$ \\
\hline \multirow[t]{5}{*}{ Population totale } & Très faible & $4 \%$ & $31 \%$ & $29 \%$ & $36 \%$ \\
\hline & Faible & $5 \%$ & $20 \%$ & $32 \%$ & $42 \%$ \\
\hline & Moyen & $9 \%$ & $13 \%$ & $31 \%$ & $48 \%$ \\
\hline & Élevé & $12 \%$ & $11 \%$ & $28 \%$ & $49 \%$ \\
\hline & Tous & $4 \%$ & $27 \%$ & $30 \%$ & $39 \%$ \\
\hline \multirow[t]{5}{*}{ Urbain } & Très faible & $4 \%$ & $24 \%$ & $35 \%$ & $38 \%$ \\
\hline & Faible & $6 \%$ & $18 \%$ & $33 \%$ & $44 \%$ \\
\hline & Moyen & $9 \%$ & $13 \%$ & $31 \%$ & $47 \%$ \\
\hline & Élevé & $12 \%$ & $11 \%$ & $28 \%$ & $49 \%$ \\
\hline & Tous & $5 \%$ & $20 \%$ & $33 \%$ & $41 \%$ \\
\hline \multirow[t]{5}{*}{ Rural } & Très faible & $3 \%$ & $37 \%$ & $25 \%$ & $35 \%$ \\
\hline & Faible & $5 \%$ & $25 \%$ & $31 \%$ & $39 \%$ \\
\hline & Moyen & $7 \%$ & $14 \%$ & $29 \%$ & $50 \%$ \\
\hline & Élevé & $12 \%$ & $19 \%$ & $30 \%$ & $38 \%$ \\
\hline & Tous & $4 \%$ & $34 \%$ & $26 \%$ & $36 \%$ \\
\hline
\end{tabular}

Notes :

*Les classes de revenu:

- Très faible-en-dessous de 2.97 USD per capita/jour

- Faible-entre 2.97 et 8.44 USD per capita/jour

- Moyen—entre 8.44 et 23.03 USD per capita/jour

- Élevé - au-dessus de 23.03 USD per capita/jour

**Toutes céréales sous forme de graine, farine ou semoule, incluant des produits amylacés exigeant un certain niveau de transformation (ex. : manioc).

***Les produits transformés comprennent : produits laitiers ; huiles alimentaires ; pains, biscuits, pâtes alimentaires ; viandes, volailles (fraîches,

congelées ou emballées) ; poissons et produits de la mer (séchés, fumés ou emballés) ; confiserie et chocolats ; produits à base de fruits et légumes conditionnés (confitures, miel, concentrés). Les produits céréaliers sous forme de farine ou semoule ne sont pas pris en compte ici dans les produits transformés car les données ne permettent pas de les distinguer des produits bruts.

Source : Banque mondiale, 2015 et calculs des auteurs

la somme monétaire de ces consommations. Celle-ci atteindrait 178 milliards USD en 2010, soit 36 \% du PIB régional. La part des dépenses totales des ménages consacrée à l'alimentation est estimée à $52 \%$ pour l'Afrique de l'Ouest en $2010^{2}$.

Le Nigéria constituerait $71.4 \%$ de ce total (Graphique 1.2). Loin derrière apparaissent le Ghana (8.5 \%), la Côte d'Ivoire (3.8 \%) puis le Sénégal (3.4\%). La surreprésentation du Nigéria, relativement à la taille de sa population (51 \% de la population régionale), est imputable à des revenus par tête plus élevés et une structure de consommation différente ${ }^{3}$. Le Nigéria produit par ailleurs 69 \% du PIB agricole régional.

L'économie alimentaire, qui comprend l'autoconsommation des ménages, se partage à peu près équitablement entre urbains (55 \%) et ruraux (45\%), alors que la région n'affiche qu'un niveau d'urbanisation de $42 \%$ en 2010. Cela implique que les urbains dépensent environ 50 \% de plus par tête que les ruraux pour leur alimentation.

\footnotetext{
2 Les données sont disponibles pour toute la zone d'étude couverte par le CSAO/OCDE (Bénin, Burkina Faso, Cabo Verde, Côte d'Ivoire, Gambie, Ghana, Guinée, Guinée-Bissau, Libéria, Mali, Mauritanie, Niger, Nigéria, Sénégal, Sierra Leone, Tchad, Togo) à l'exception de la Guinée-Bissau.

3 Les fruits et légumes représentent $38 \%$ des consommations alimentaires des ménages nigérians contre $23 \%$ pour le reste de l'Afrique de l'Ouest. Parallèlement, en 2010, 68 \% des légumes de la région sont produits au Nigéria (FAOSTAT, 2015).
} 


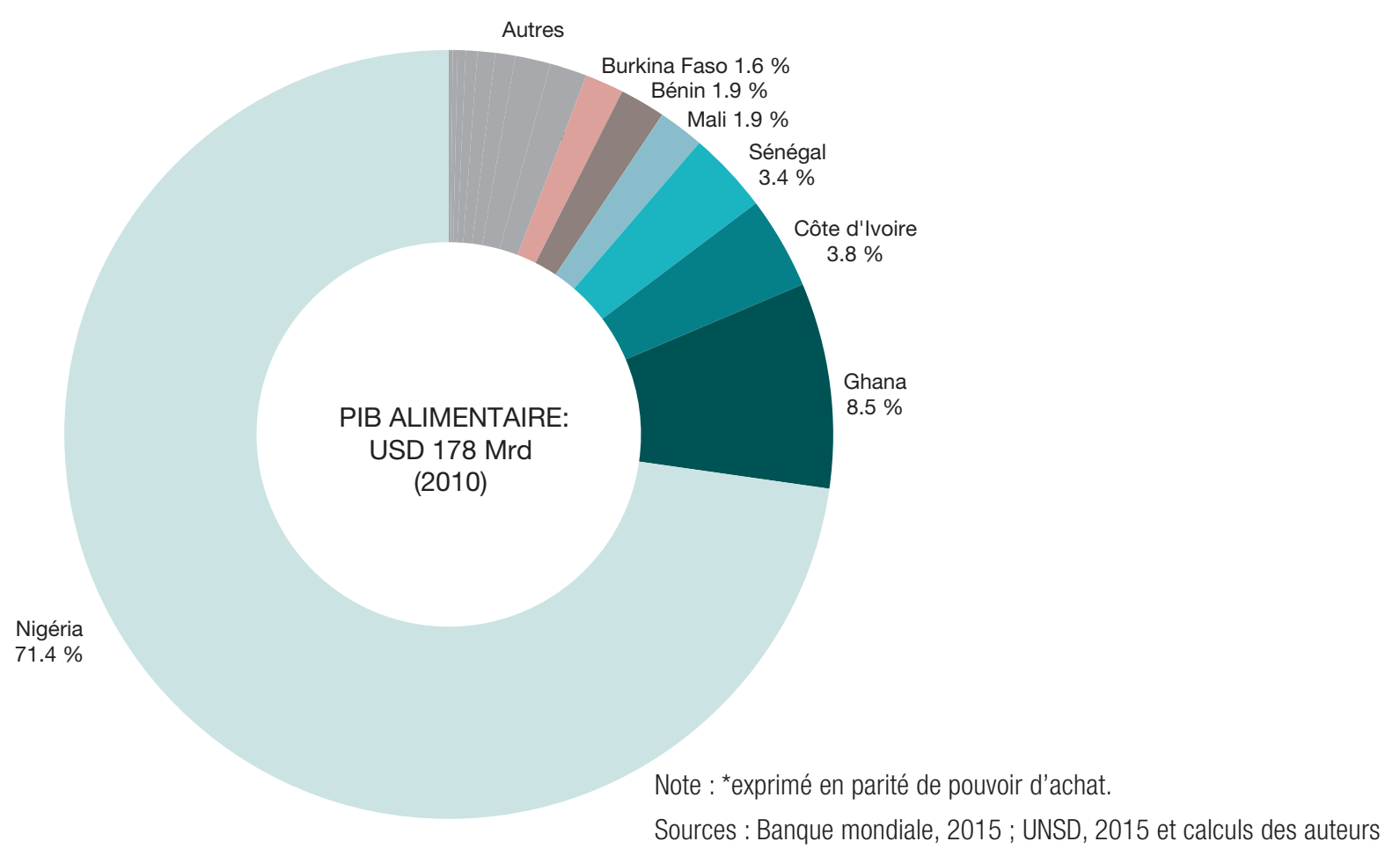

En 2010, la population se répartit équitablement entre producteurs et non-producteurs agricoles au niveau régional (OCDE, 2013). Si l'autoconsommation peut être estimée à environ $60 \%$ des consommations alimentaires des ménages agricoles ${ }^{4}$ et $10 \%$ pour les ménages non agricoles, alors deux tiers des consommations alimentaires ont fait l'objet d'échanges sur les marchés. Ces transactions commerciales représentent donc un total de 126 milliards USD en 2010. Elles impliquent la réalisation d'une succession d'activités et la participation de nombreux acteurs différents ; leur montant révèle l'importance de ces nouvelles activités et l'ampleur des mutations traversées par l'Afrique de l'Ouest. Celle-ci est passée d’une économie essentiellement de subsistance à une économie intégrée au marché. La taille du marché alimentaire montre par ailleurs la faible dépendance de l'Afrique de l'Ouest aux importations alimentaires. Celles-ci ont certes augmenté, mais ne sont estimées qu'à 12 milliards USD en 2010, soit $6.5 \%$ de la demande intérieure (FAOSTAT, 2015).

L'agriculture ouest-africaine a massivement redirigé ses productions vers son marché régional de produits vivriers. L'ensemble des consommateurs exclusivement dépendants des marchés pour leurs approvisionnements alimentaires, représente un marché, en volume, de 143 billions kcal

\section{Les transactions alimentaires représentent un total de 126 milliards USD en 2010.} en 2010. Ce chiffre a été multiplié par 13 en 60 ans $^{5}$. En 1961, au lendemain des indépendances, les exportations agricoles extra-régionales étaient aussi importantes que ce marché intérieur de consommateurs non-producteurs. Aujourd'hui, elles ne représentent plus que $12 \%$ de la totalité de cette demande (Graphique 1.3). Les économies alimentaires ouest-africaines s'éloignent donc de plus en plus des modes d'échanges agricoles hérités de l'époque des

\footnotetext{
4 Compte tenu d'un niveau d'autoconsommation estimé à $50 \%$ pour les ménages ruraux (Bricas et Tchamda, 2015) et $25 \%$ de ménages ruraux non agricoles (OCDE, 2013), ainsi qu'un niveau d'autoconsommation de $10 \%$ pour les ménages urbains.

5 Le suivi de l'évolution en volume des quantités consommées permet de faire abstraction des prix et le choix de prendre les kilocalories (kcal) comme étalon de mesure, autorise les comparaisons de niveaux de consommation et taille des marchés entre pays ou à différentes époques.
} 
indépendances. D'une part, les échanges locaux liés à l'autosubsistance des communautés villageoises laissent place à un commerce à plus longue distance rapprochant villes et campagnes ou intra-régional. D’autre part, les exportations outre-mer de produits de rente représentent une proportion désormais bien faible comparativement aux échanges sur le marché régional. Les opportunités économiques du marché alimentaire régional dominent désormais.

\section{Graphique 1.3}

Exportations agricoles et marché régional - Afrique de l'Ouest (1961-2010)

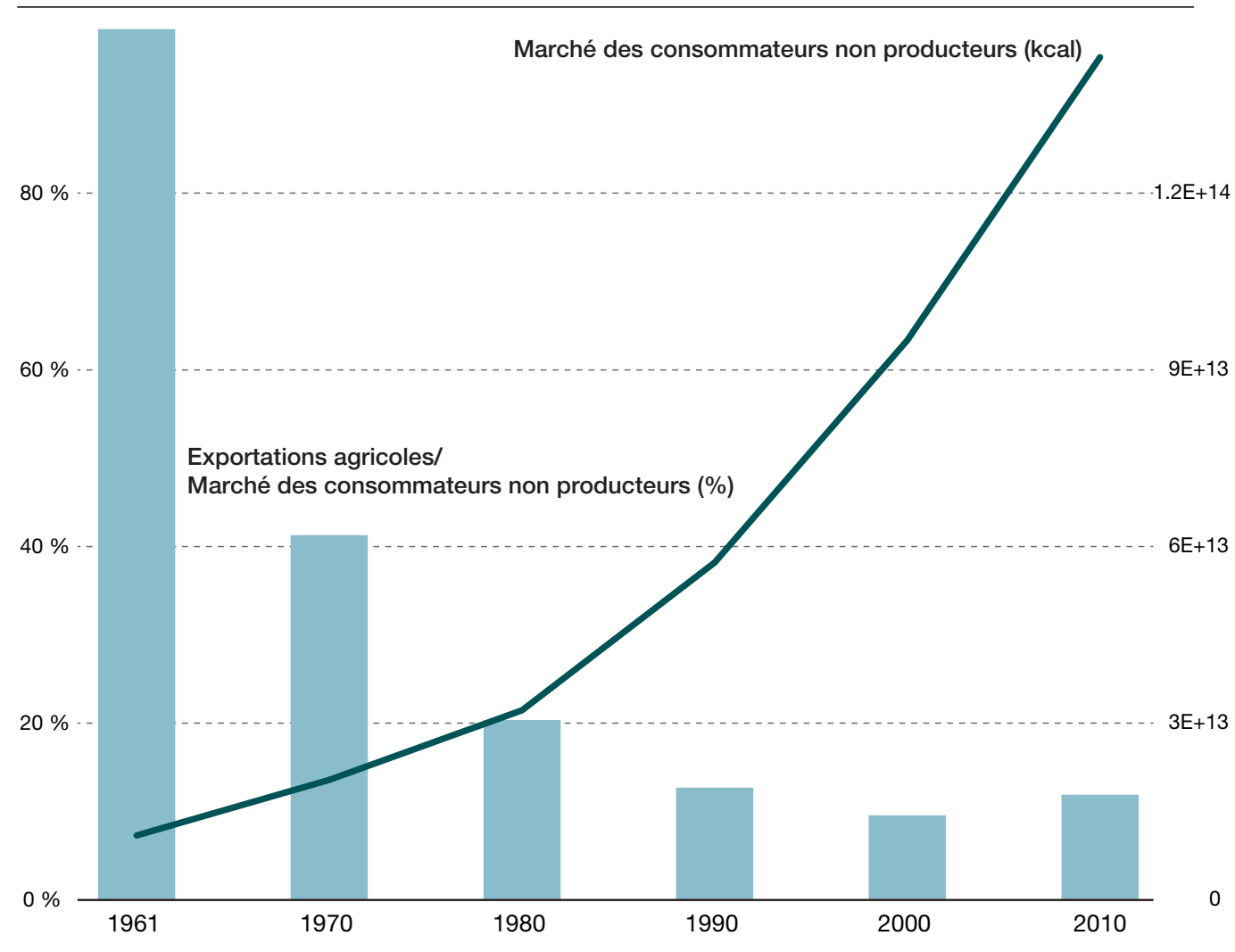

Source : FAOSTAT, 2015 et calculs des auteurs

\section{UNE ÉCONOMIE ALIMENTAIRE DE MOINS EN MOINS AGRICOLE}

Le mouvement d'extension du marché offre de nouvelles opportunités en matière de création de valeur ajoutée et diversification des activités dans l'agro-alimentaire. Les activités économiques en amont (intrants et services) et en aval (commercialisation et transformation) de la production agricole prennent leur essor. Ces nouvelles activités, non agricoles, contribuent à hauteur de 40 \% de la valeur ajoutée de l'économie alimentaire.

\subsection{Création de valeur ajoutée au-delà de l'agriculture}

L'augmentation et l'évolution de la structure des dépenses alimentaires induisent une modification du partage de valeur ajoutée entre l'agriculture et les autres secteurs de l'économie alimentaire. Les activités post-récolte, du transport à la distribution en passant par la transformation alimentaire, augmentent en importance relativement à l'agriculture.

Le rapport entre la valeur monétaire de l'économie alimentaire et le PIB agricole, qui ne comprend pas que la production de denrées agricoles à destination de l'alimentation humaine (coton, bois, etc.), donne une approximation de la diversification des activités agro-alimentaires. Il indique le degré de développement des activités connexes à 
l'agriculture et permet de saisir le niveau de transformation structurelle dans le secteur agro-alimentaire. Ce ratio s'établit à 1.6 au niveau régional, indiquant que l'économie alimentaire est 60 \% plus importante que la valeur du PIB agricole (Tableau 2.1). Afin de faciliter la comparaison entre une économie alimentaire à destination du marché local et une production agricole qui approvisionne également les marchés internationaux, les exportations agricoles ont ici été soustraites au PIB agricole. Étant donné qu'une part non négligeable du PIB agricole n'est pas destinée à l'alimentation humaine ${ }^{6}$, la part de l'agriculture dans l'économie alimentaire ne dépasserait pas $60 \%$. Autrement dit, $40 \%$ de la valeur ajoutée de l'économie alimentaire n'est plus le fait de l'agriculture.

$40 \%$ de la valeur ajoutée de l'économie alimentaire n'est plus le fait de l'agriculture.
Le ratio est supérieur à 1 pour les pays qui affichent également les niveaux de PIB par tête et/ou les volumes d'importations alimentaires les plus importants, comme le Cabo Verde, le Ghana, le Nigéria ou le Sénégal (Tableau 2.1). Afin d'évaluer la contribution de l'offre domestique à l'alimentation des ménages ouest-africains, les importations alimentaires peuvent être soustraites au PIB alimentaire. Ainsi corrigé, le rapport s'établirait à 1.5 au niveau régional ; ce qui indique que la majeure partie de la demande régionale est satisfaite par l'offre locale. Ces résultats révèlent qu'un processus de sophistication des chaînes de valeur alimentaires est à l'œuvre et que les activités post-récolte se développent. Bien que les données manquent pour caractériser précisément les évolutions de la structure de l'économie alimentaire, l'importance des activités non agricoles observée en 2010 suggère qu'on assiste à une recomposition sectorielle, avec une baisse de la part de l'agriculture dans l'économie alimentaire et un déplacement des activités économiques vers le secteur agro-industriel et les services.

Tableau 2.1

Part de l'économie alimentaire par rapport à l'activité agricole (2010)

\begin{tabular}{lcccc}
\hline \hline & $\begin{array}{c}{[1]} \\
\text { PIB ALIM/PIB }\end{array}$ & $\begin{array}{c}{[2]} \\
\text { PIB AGRI*/PIB }\end{array}$ & $\begin{array}{c}\text { [1]/[2] } \\
\text { PIB ALIM/PIB AGRI* }\end{array}$ & $\begin{array}{c}\text { [3] } \\
\text { IMPORT ALIM/PIB AGRI* }\end{array}$ \\
\hline Cabo Verde & $31 \%$ & $8 \%$ & 3.9 & 1.3 \\
Sénégal & $41 \%$ & $13 \%$ & 3.2 & 0.7 \\
Bénin & $43 \%$ & $23 \%$ & 1.8 & 0.3 \\
Guinée & $39 \%$ & $22 \%$ & 1.7 & 0.3 \\
Nigéria & $37 \%$ & $23 \%$ & 1.6 & 0.1 \\
Afrique de l'Ouest & $36 \%$ & $23 \%$ & 1.6 & 0.1 \\
Ghana & $35 \%$ & $24 \%$ & 1.5 & 0.1 \\
Mauritanie & $29 \%$ & $19 \%$ & 1.5 & 0.4 \\
Gambie & $36 \%$ & $26 \%$ & 1.4 & 0.6 \\
Libéria & $67 \%$ & $46 \%$ & 1.4 & 0.5 \\
Guinée-Bissau & $49 \%$ & $38 \%$ & 1.3 & 0.2 \\
Togo & $39 \%$ & $32 \%$ & 1.2 & 0.2 \\
Niger & $38 \%$ & $38 \%$ & 1 & 0.1 \\
Tchad & $18 \%$ & $21 \%$ & 0.9 & 0.1 \\
Mali & $31 \%$ & $34 \%$ & 0.9 & 0.1 \\
Sierra Leone & $40 \%$ & $52 \%$ & 0.8 & 0.1 \\
Burkina Faso & $23 \%$ & $28 \%$ & 0.8 & 0.1 \\
Côte d'Ivoire & $28 \%$ & $n / a$ & & $n$ \\
\hline
\end{tabular}

Note : *PIB agricole ajusté : PIB agricole auquel les exportations agricoles ont été soustraites.

Sources : FAOSTAT, 2015 ; UNSD, 2015 et calculs des auteurs

6 Les seules activités forestières peuvent être estimées à environ $8 \%$ du PIB agricole en Afrique sub-saharienne en 2010 (FAOSTAT). Par ailleurs, les auteurs du rapport Agrimonde estiment en Afrique sub-saharienne à, respectivement, $5 \%$ et $2 \%$ les parts des calories alimentaires végétales destinées à des valorisations agricoles non alimentaires (VANA) et aux semences, auxquelles il faut ajouter environ $10 \%$ de pertes (Paillard et al., 2010). 
Encadré 2.1

Chaînes de valeur alimentaires, agribusiness et agro-industries - définitions et concepts

L'Organisation des Nations Unies pour le développement industriel (ONUDI), qui utilise les termes en anglais d'agro-food value chain, définit les chaînes de valeur comme l'ensemble des « acteurs connectés le long d'une chaîne produisant et délivrant des biens et des services à des consommateurs finaux au travers une séquence complexe d'activités ${ }^{1}$ (Henriksen et al., 2010).

En français, cette définition rapproche le concept de « chaîne de valeur » de la notion de « filière ». Des différences subtiles existent cependant entre les deux termes ; alors que les « chaînes de valeur » mettent l'accent sur l'analyse de la création de valeur ajoutée et de la compétitivité dans certaines activités, les « filières » visent plus à étudier les relations entre les activités dans la suite d'opérations. En d'autres termes, alors qu'une analyse " chaîne de valeur » serait plus sectorielle et spécifique, l'analyse filière renverrait plus à la notion d'ensemble et serait plus systémique. Dans la pratique, les deux termes apparaissent interchangeables.

La FAO a proposé une définition des chaînes de valeur agricoles comme l'ensemble «des acteurs et des activités qui font passer un produit agricole de base du stade de la production dans les champs à sa consommation finale, processus dont chaque stade voit de la valeur être ajoutée au produit. Une chaîne de valeur peut être un lien vertical ou un réseau entre diverses organisations d'entrepreneurs indépendantes et peut concerner la transformation, l'emballage, l'entreposage, le transport et la distribution. » (FAO, 2010). Les termes « chaîne de valeur agricole » ou " chaîne de valeur agro-alimentaire », en anglais " agricultural value chain » et " agro-food value chain », sont considérés similaires. Les chaînes de valeur alimentaires peuvent alors être entendues comme la séquence d'activités dont l'objectif final est la seule alimentation humaine.

Par ailleurs, l'agribusiness est communément défini comme la « part de l'économie moderne dédiée à la production, transformation et distribution des produits alimentaires, fibreux et autres produits dérivés, y compris les institutions financières qui financent ces activités ${ }^{2}$ (Encyclopaedia Britannica, 2011). II englobe i) les producteurs d'intrants à destination de la production agricole (semences, engrais, etc.) ; ii) les exploitations agricoles commerciales ; iii) l'agro-industrie, qui inclut le sous-secteur Alimentation, boissons et tabac (ABT) mais également le travail des textiles, cuir, bois, etc. ; iv) les fabricants de matériel pour la transformation des matières premières agricoles ; v) et enfin les services, du financement au marketing, en passant par la distribution, le transport et le conditionnement (BAD, 2014).

De leur côté, les agro-industries sont le « sous-ensemble du secteur manufacturier qui transforme les produits bruts et intermédiaires qui proviennent de l'agriculture, de la pêche et des activités forestières » ${ }^{3}$ (Henson et Cranfield, 2009). Le graphique 2.1 propose une représentation schématique de ces différents concepts. »

1 Traduction des auteurs : "Actors connected along a chain producing and bringing goods and services to end consumers through a complex and sequenced set of activities ".

2 Traduction des auteurs: "Part of a modern economy devoted to the production, processing and distribution of food, fibre products and by-products including the financial institutions that fund these activities"

3 Traduction des auteurs: "Subset of the manufacturing sector that processes raw materials and intermediate products derived from agriculture, fisheries and forestry".

\section{Graphique 2.1}

Chaînes de valeur alimentaires, agribusiness et agro-industries

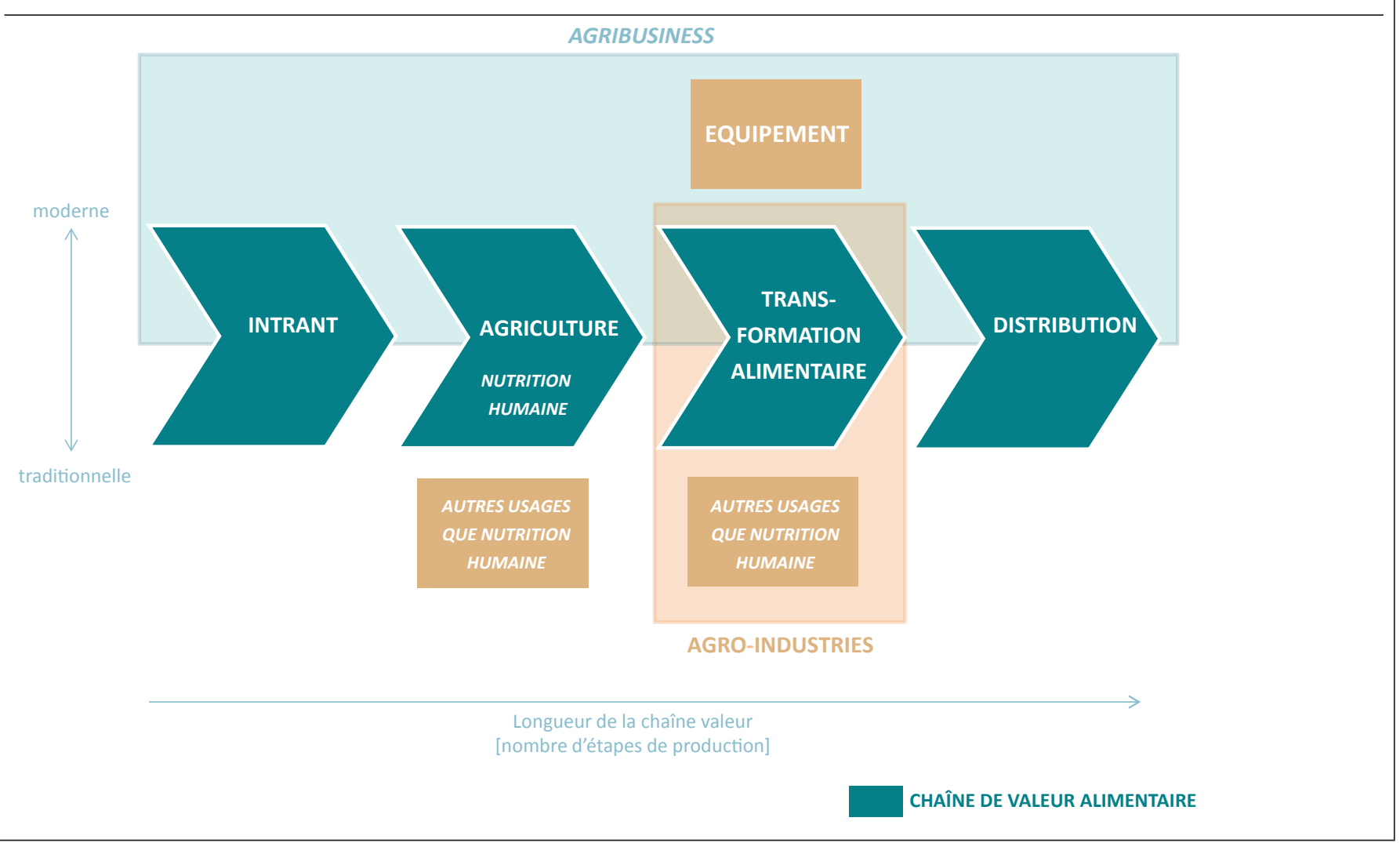




\subsection{Le développement des chaînes de valeur alimentaires}

De nouveaux acteurs entrent dans l'économie alimentaire avec le développement d'activités hors agriculture, en particulier dans les segments post-récolte. Les formes d'organisation et la longueur des filières sont variées et dépendent des caractéristiques des biens échangés. Dans le cas des produits périssables, comme les légumes et les fruits, les circuits sont courts avec de zéro à un intermédiaire entre producteurs et consommateurs. En revanche, les produits transformés à base de céréales, qu'il s'agisse des produits ( prêts à la consommation » (pains, galettes, gâteaux, etc.) ou (c prêts à l'emploi ») (farines, semoules, granules, etc.), passent par plusieurs étapes avant le consommateur final. Ils font l'objet de procédés plus ou moins sophistiqués de transformation, stabilisation et conditionnement. La matière première a dû être ensachée, transportée, déchargée, stockée, contrôlée, calibrée, nettoyée, broyée, laminée, parfois séchée ou grillée, congelée ou réfrigérée, conditionnée, emballée, etc. Certaines de ces opérations se répètent le long de la chaîne logistique. De manière générale, l'extension des marchés et la demande pour des produits plus transformés impliquent le développement de ces activités post-récolte qui ne relèvent pas du secteur primaire et s'effectuent de plus en plus hors de l'exploitation agricole et du ménage. Elles peuvent être schématiquement regroupées dans les deux grands segments de la transformation alimentaire et de la distribution le long des chaînes de valeur (Encadré 2.1).

Le développement des activités de transformation alimentaire est récent. Celles-ci sont difficiles à comptabiliser, notamment en raison de leur caractère informel. Ce domaine recouvre en effet une grande diversité de métiers et d'acteurs, à des niveaux différents d'avancement en termes d'utilisation des technologies. À côté de quelques structures industrielles relativement larges (brasseries, minoteries, etc.), le secteur est essentiellement constitué par un tissu de micro-entreprises et de PME, souvent familiales et informelles. Les procédés de production sont souvent artisanaux, peu mécanisés et standardisés. Ces entreprises peuvent se transformer jusqu'à s'industrialiser, pour ravitailler les marchés.

Définie comme un sous-ensemble du secteur manufacturier qui transforme les produits bruts issus de l'agriculture, l'agro-industrie inclut notamment les activités de transformation alimentaire. Les quelques sources de données disponibles révèlent que son volume global d'activités est en progression depuis les années 80. Au Sénégal, l'agroindustrie constitue le premier sous-secteur manufacturier et progresse de $4 \%$ par an depuis 1980 (Graphique 2.2).

Graphique 2.2

Volume des activités manufacturières au Sénégal (1980-2010)

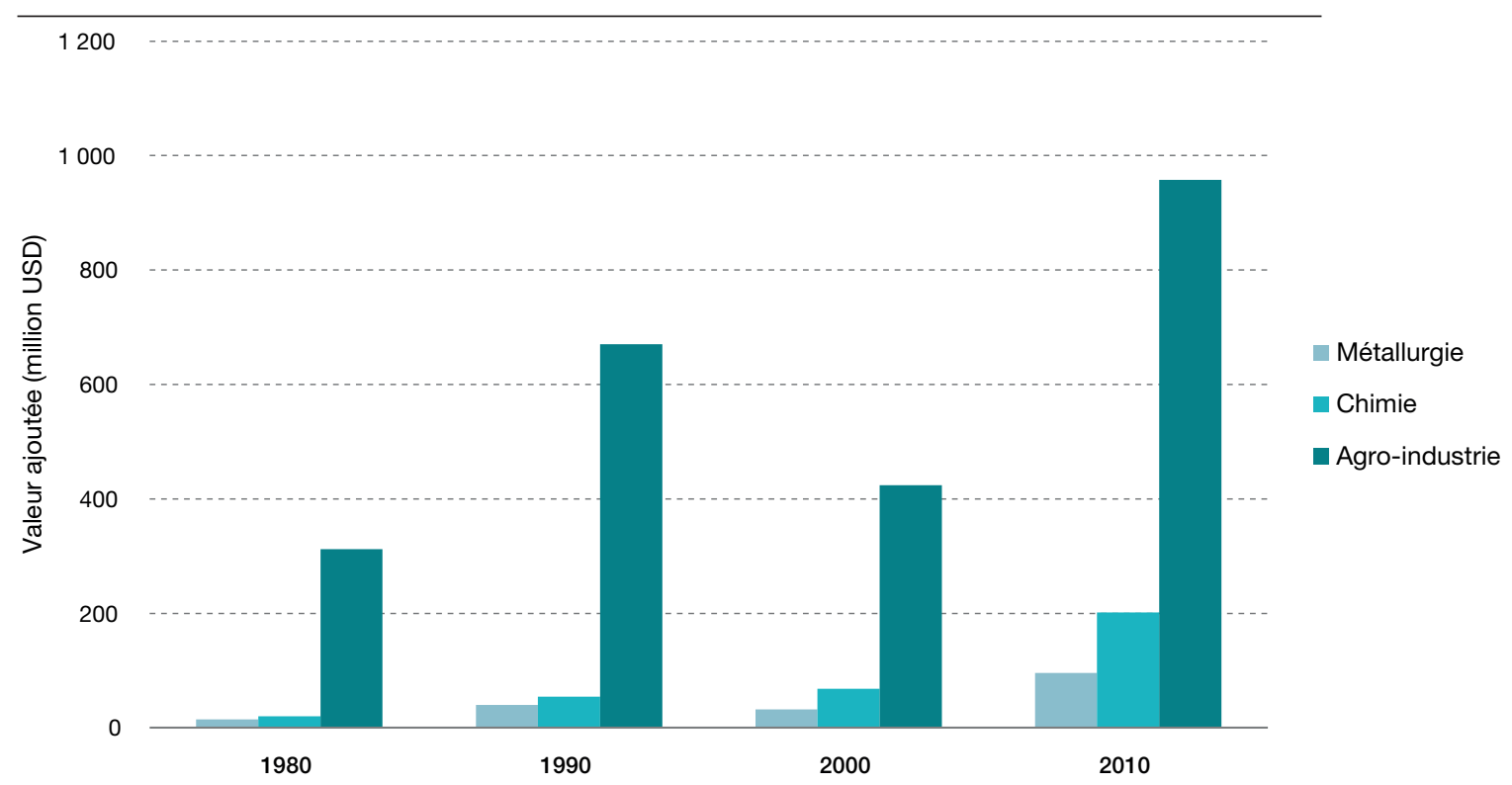

Sources : ANSD Sénégal, 2015 et calculs des auteurs 


\section{Encadré 2.2}

Participation de l'Afrique de l'Ouest dans les chaînes de valeur alimentaires mondiales

L'éclatement géographique des processus de production s'observe également à l'échelle internationale. Aujourd'hui, les entreprises agroalimentaires ont la possibilité de répartir les activités de production, de transformation et de distribution dans le monde entier en raison d'une réduction considérable du coût des échanges. Le fait que ces activités soient réparties de manière croissante entre différents pays explique que I'on parle de Chaine de Valeur «Mondiale» (CVM).

L'analyse des indicateurs de participation révèle que les pays d'Afrique de l'Ouest sont connectés aux CVMs alimentaires, mais n'y contribuent pas tous de la même manière. Le Ghana et la Côte d'Ivoire, par exemple, se positionnent davantage en tant qu'acheteurs de produits intermédiaires (participation « en aval » ou Backward), tandis que le Nigeria est en premier lieu fournisseur de produits intermédiaires (participation « en amont » ou Forward). La taille du marché intérieur est l'un des principaux facteurs permettant d'expliquer cette différence (OCDE, 2016). Le Nigéria peut compter sur son marché pour s'approvisionner en intrants (ce qui tend à réduire son engagement " en aval »), ainsi que pour produire et exporter des produits intermédiaires (ce qui tend à accroitre sa participation « en amont »).

\section{Graphique 2.3}

Participation aux chaînes de valeur alimentaires mondiales (2011)

\section{Indicateur Backward*}

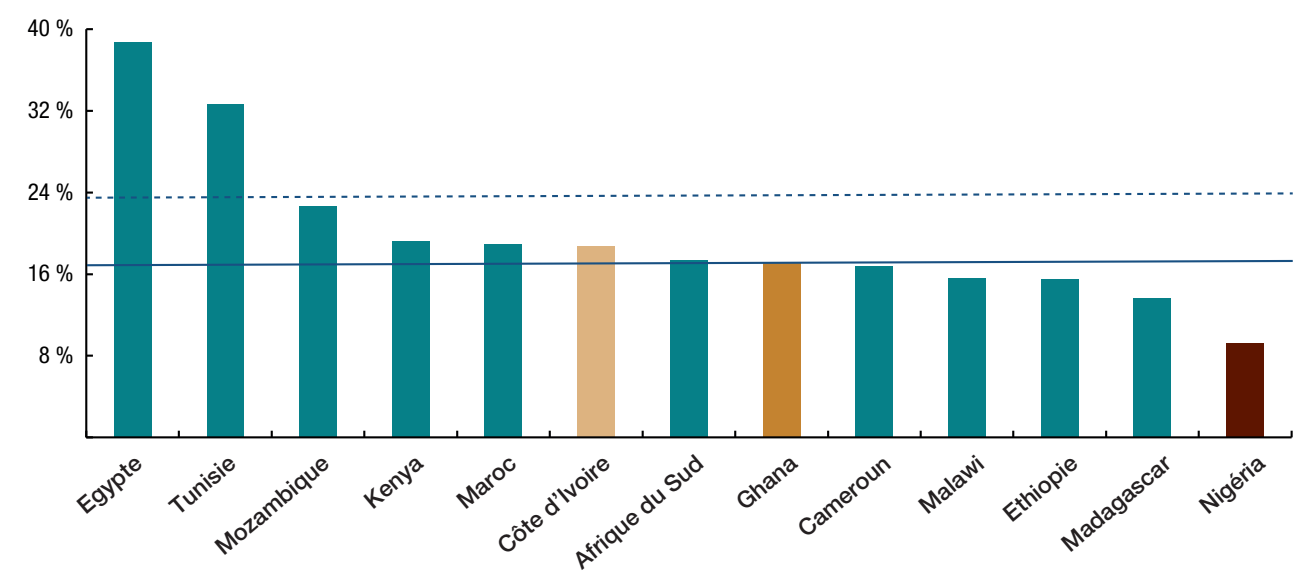

Indicateur Forward**

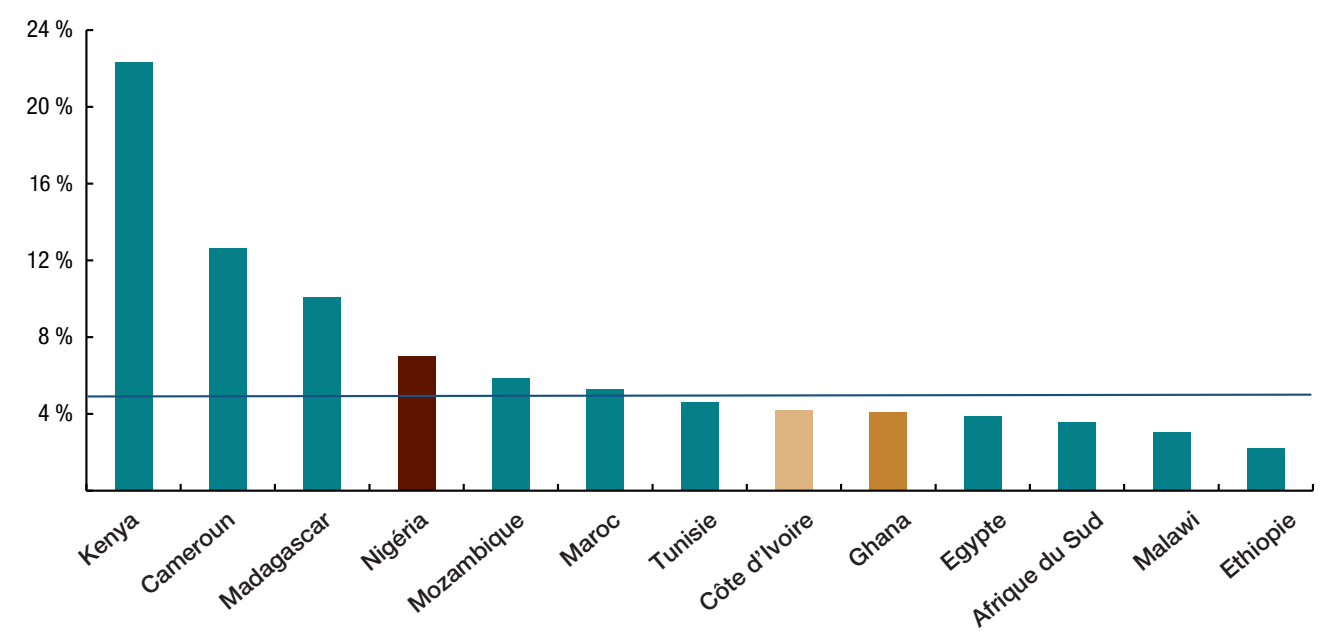

Notes :

*L'indicateur Backward est égal à la somme des importations en valeur ajoutée de produits intermédiaires incorporées dans les exportations divisée par les exportations brutes.

**L'indicateur Forward est égal à la somme des exportations en valeur ajoutée utilisées comme intrants dans les exportations du pays partenaire, divisée par les exportations brutes.

Pour l'indicateur Backward, la ligne continue indique la moyenne pour les pays en développement, la ligne discontinue celle pour les pays développés. Pour l'indicateur Forward, la moyenne est la même pour les deux types d'économies. Les données couvrent 20 produits alimentaires et les 70 pays disponibles dans la base de données GTAP dont 13 pays africains. 
Les industries de transformation alimentaire, qui constituent une composante des agro-industries, progressent de plus de $7 \%$ par an depuis 2000 au Sénégal et contribuent à hauteur de 37 \% à la création de valeur ajoutée provenant de l'industrie manufacturière en 2010. Les données manquent cependant pour suivre plus précisément les changements structurels à l'œuvre dans le secteur agro-alimentaire.

Les réseaux de distribution sont, de leur côté, composés de collecteurs, grossistes, transporteurs et détaillants. Ces derniers opèrent sur les marchés traditionnels, disposent parfois d'échoppes et magasins, voire de supermarchés en milieu urbain, mais incluent également une myriade de vendeurs ambulants. Le rôle du secteur informel est extrêmement important dans la commercialisation des denrées alimentaires. La restauration de rue se développe également, notamment en raison de la demande importante de collations ( ( prêtes à la consommation ) de la part des travailleurs urbains. Ces réseaux de distribution sont essentiels au bon fonctionnement des marchés et à l'accessibilité des ménages à l'alimentation.

L'urbanisation, et l'augmentation et la concentration de la demande alimentaire qui l'accompagnent, impose de penser les circuits d'approvisionnement alimentaire dans une logique spatiale. À mesure que zones de production et zones de consommation se distinguent, la gestion de l'espace et de ses connexions devient une condition de la sécurité alimentaire. On observe non seulement la cohabitation d'un plus grand nombre d'activités et d'acteurs économiques dans les chaînes de valeur, mais celles-ci s'inscrivent également dans un espace géographique plus étendu qui exige de penser des circuits de distribution plus élaborés.

\section{IMPLICATIONS POUR LES POLITIQUES ET LA SÉCURITÉ ALIMENTAIRE}

La population et l'urbanisation vont continuer de croître; les transformations et la croissance de l'économie alimentaire corrélées vont s'accélérer. Les tendances actuelles montrent que la demande croîtra plus rapidement pour les biens transformés et/ou à haute valeur ajoutée. La plus grande part de cette croissance se fera hors agriculture, dans les segments de la transformation, distribution et commercialisation des aliments. Ces activités contribueront à la création de valeur ajoutée et d'emplois, en milieux urbain et rural.

Ces mutations accentuent la complexité du système alimentaire et donc des politiques. Les chaînes de valeur alimentaire impliquent plus d'acteurs dans un espace plus étendu. Les nouvelles activités impliquent également de nouveaux besoins, en termes de nouvelles technologies

Les segments post-récolte des chaînes de valeur alimentaires connaîtront la croissance la plus forte. et compétences, de développement d'infrastructures, de financement et de coordination.

Au niveau des politiques de sécurité alimentaire et nutritionnelle, les segments aval des chaînes de valeur doivent faire partie de la grille de suivi et d'analyse. Il est nécessaire de développer des capacités ciblées aux contraintes des populations vulnérables urbaines et de suivre les nouveaux enjeux de santé publique liés à la transformation des habitudes de consommation.

\subsection{Connecter et coordonner les activités de l'économie alimentaire}

Le marché est désormais essentiel à l'alimentation des ménages ouest-africains. Ce passage à des logiques commerciales est à l'origine de mutations profondes de l'économie alimentaire. Les nouvelles activités de transformation, de logistique et de distribution sont les segments des chaînes de valeur alimentaires qui connaîtront la croissance la plus forte sur les prochaines décennies. L'étude AGWA (Hollinger et Staatz, 2015) rapporte en effet des élasticités revenu de la demande plus élevées et supérieures à 1 pour un certain 
nombre de produits transformés courants (Graphique 3.1) ; ceci indique que la demande pour ces produits croîtra plus rapidement que l'augmentation des revenus. De nouveaux acteurs et activités vont entrer dans cette économie alimentaire pour répondre à cette demande. Leur

À mesure que l'économie alimentaire augmente, des progrès en matière de structuration des filières sont nécessaires. coordination s'appuie aujourd'hui sur une combinaison de mécanismes de prix et d'appartenance à des réseaux, entendus comme système de relations. À mesure que le volume de cette économie alimentaire augmente, des progrès en matière de structuration des filières et de mise en relation des différents maillons de la chaîne sont nécessaires. Il importe de faciliter le développement de ces activités et de fluidifier la circulation de l'information entre les différentes parties prenantes.

Graphique 3.1

Élasticités revenu de la demande en milieu urbain - 8 pays en Afrique de l'Ouest*

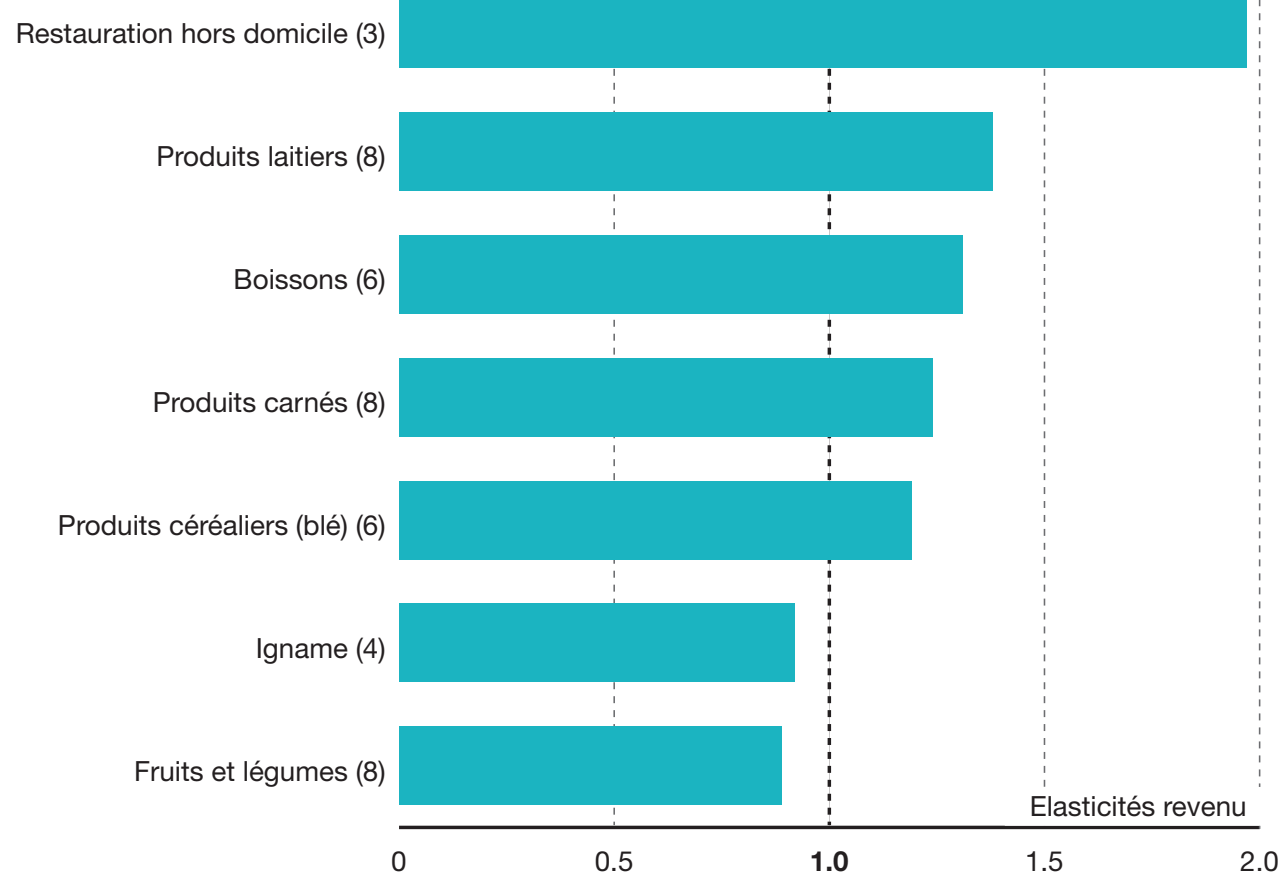

Note : *Burkina Faso, Côte d’Ivoire, Ghana, Mali, Niger, Nigéria, Sénégal, Togo. Entre parenthèses, le nombre de pays pour lesquels les élasticités revenu étaient disponibles.

Source : Hollinger et Staatz, 2015

L'accroissement de la demande et les évolutions des habitudes de consommation alimentaire créent de nouveaux débouchés pour la production agricole. Celle-ci augmente sous l'effet de la demande et se diversifie vers de nouveaux produits. Ces transformations exigent notamment de revoir les filières jugées stratégiques. Le développement des chaînes de valeur alimentaires contribue également aux progrès du secteur agricole par les recommandations techniques qu'elles imposent sur la production et les services offerts. Une chaîne d'approvisionnement plus longue requiert que les producteurs et autres intervenants répondent à des standards de qualité plus exigeants, notamment afin d'assurer la salubrité des aliments. Les agro-industries exigent par ailleurs souvent des propriétés très précises (forme, taille, poids, texture, teneur en vitamines, etc.). Les normes et standards jouent un rôle fondamental dans la transmission des informations sur la nature des produits et les conditions de production, transformation et transport. À ce titre, ils participent à l'organisation et la coordination des acteurs des chaînes de valeur. In fine, 
ils permettent la réalisation de gains d'efficacité, en réduisant les coûts de transaction, et sont nécessaires à la massification de la production alimentaire. Le défi reste de penser l'établissement de normes et standards appropriés à une agriculture encore à majorité familiale.

Par ailleurs, ces évolutions exigent de nouvelles compétences au sein des filières, tant sur le plan technique que de la gestion de l'entreprise. Il existe un réel besoin en termes d'éducation et de formation professionnelle plus approfondies, ainsi qu'en services de conseil appropriés pour accompagner ces nouvelles branches d'activités.

Le développement des chaînes de valeur s'inscrit également dans un espace géographique plus étendu. L'augmentation et la concentration de la demande alimentaire dans les villes, ainsi que la distinction plus forte entre zones de production et consommation, imposent de penser une gestion de l'espace et de ses connexions. Les infrastructures de transport, stockage et communication, qui facilitent la mobilité et les échanges, contribuent à modeler l'espace marchand. Les infrastructures routières, en particulier, jouent un rôle structurant dans l'organisation des échanges. Elles contribuent au désenclavement des zones de production, facilitant l'accès aux intrants agricoles et les mises en marché.

Le développement des activités de l'économie alimentaire appelle d'importants investissements. Les parties prenantes du système alimentaire sont essentiellement des acteurs privés avec un accès difficile aux financements. Des mécanismes incitatifs et des systèmes de garantie doivent être pensés en sus de l'investissement public. Un climat d'affaires favorable est nécessaire pour développer l'investissement privé.

\section{Améliorer la compétitivité, profiter des opportunités}

Les activités post-récolte concourent également à la formation des prix alimentaires. Elles contribuent à l'augmentation croissante de la valeur des produits alimentaires et impliquent une addition des coûts de transaction. Aujourd'hui, le niveau des prix alimentaires en Afrique de l'Ouest est plus élevé que dans le reste du monde. À niveau de revenu comparable, ces derniers sont supérieurs en Afrique, et particulièrement en Afrique de l'Ouest, à ceux pratiqués en Asie (Graphique 3.2).

\section{Graphique 3.2}

Indice des niveaux de prix alimentaires et PIB par tête (2011)
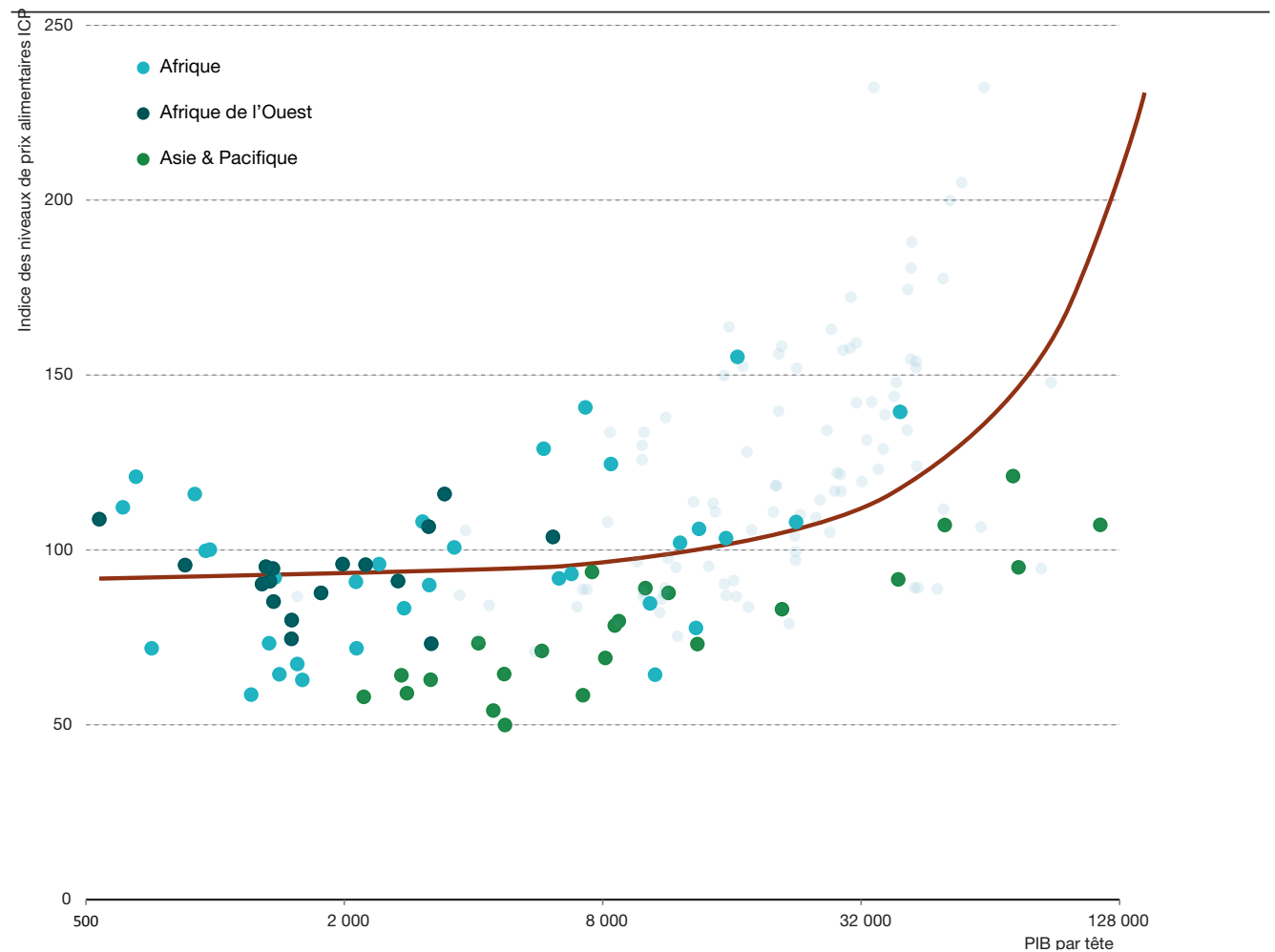

Sources : ICP 2011/Banque mondiale, 2015 et calculs des auteurs 
Une meilleure compréhension de la formation des prix alimentaires aiderait à saisir les contraintes et faiblesses le long des chaînes de valeur expliquant entre autres les prix élevés. Agir sur ces obstacles permettrait de réduire les prix pour les ménages tout en maintenant les revenus des producteurs. La faible compétitivité des chaînes alimentaires locales révélée, entre autres, par les prix élevés reste un défi en matière de développement. Les différents segments des chaînes de valeur alimentaires offrent d'importantes opportunités d'emploi. Une étude conjointe de la Banque mondiale et du Bureau international du travail montre qu'au Sénégal l'agribusiness serait le premier créateur d’emplois dans le secteur informel (81 \%) (Réseau pour l'emploi des jeunes et Fondation internationale de la jeunesse, 2009). La contribution de l'économie alimentaire aux transformations structurelles, au développement agricole et rural, aux revenus ou à la création d'emplois est cruciale. Dans un contexte où les consommateurs demandent des produits d'une valeur et qualité grandissantes, les produits ouest-africains entrent de plus en plus en concurrence avec des produits importés. Cette compétition n’est pas limitée aux produits de consommation finale, les grosses unités de transformation importent de plus en plus de matières brutes (blé, sorgho). L'amélioration nécessaire de la compétitivité des produits ouest-africains dépendra des gains réalisés à tous les stades de la chaîne de valeur.

\subsection{Adapter les outils de la sécurité alimentaire et nutritionnelle}

\section{Suivre l'ensemble du processus de production alimentaire}

Les mutations de l'économie alimentaire ont des implications importantes pour les systèmes d'information sur la sécurité alimentaire et nutritionnelle et les mécanismes de résilience.

Le fonctionnement fluide des processus de production agricole, transformation alimentaire, transport, stockage et distribution, indispensables à la mise en marché, est essentiel à la stabilité des approvisionnements alimentaires. Ces processus peuvent être confrontés à des blocages et chocs qui doivent être anticipés par les mécanismes de prévention des crises alimentaires. Les activités post-récoltes et leur suivi participent également des capacités de résilience du système alimentaire dans la mesure où elles peuvent atténuer l'ampleur d'un choc et faciliter notamment la gestion de l'information et des stocks. Les difficultés rencontrées par les acteurs des segments aval doivent donc faire explicitement partie de la grille d'analyse des facteurs contributifs pouvant avoir un impact sur la sécurité alimentaire et nutritionnelle. Ces segments concernent aujourd'hui directement les populations vulnérables dans la mesure où elles consacrent plus d'un tiers de leur budget alimentaire aux produits transformés, et qu'une part importante d'entre elles vit en ville, dépendant des circuits alimentaires marchands.

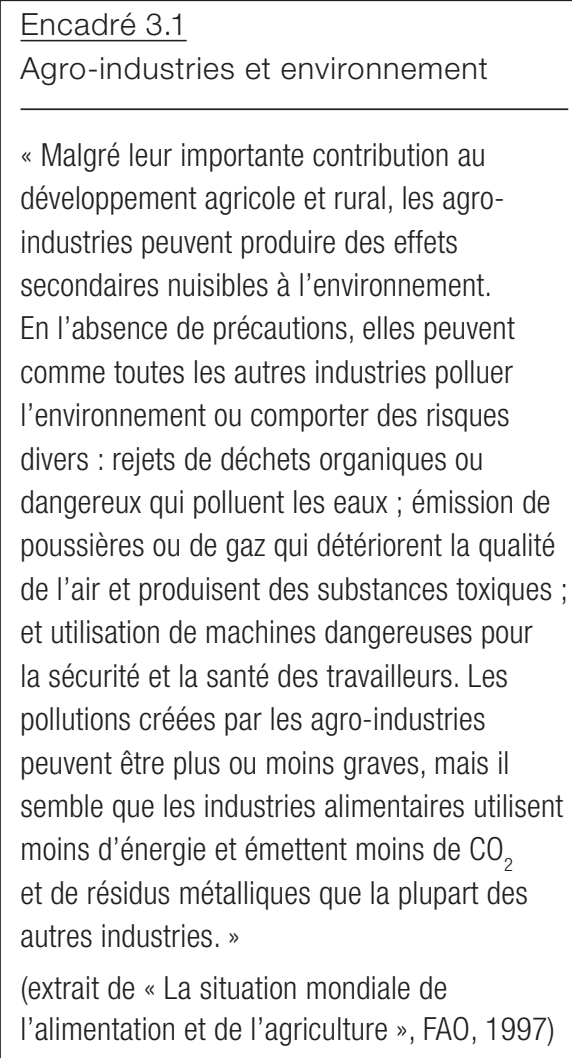

Enfin, ces activités contribuent aux coûts et participent du prix final des denrées qui, lui, influence les décisions d'allocation des ressources des ménages. Toute fluctuation des prix, liée aussi bien à la matière première qu'aux processus de transformation, peut grever le pouvoir d'achat des ménages et donc la sécurité alimentaire et nutritionnelle. Il importe donc d'inclure des produits transformés essentiels lors du suivi des prix à la consommation des produits alimentaires courants.

\section{Nouveaux enjeux de santé publique}

Les évolutions des habitudes de consommation alimentaires sont par ailleurs à l'origine de nouveaux enjeux nutritionnels et de santé publique. S'il est possible de relever une réduction de la malnutrition et une amélioration de la diversité alimentaire, la transition nutritionnelle en cours implique également une augmentation des apports en graisses et sucres raffinés 
qui peuvent avoir des répercussions négatives en santé publique. Il y a dans les pays de l'Afrique sub-saharienne, en particulier en ville, une augmentation de la prévalence de l'obésité et du surpoids, et de maladies chroniques non transmissibles, comme le diabète et les maladies cardio-vasculaires (FAO et al., 2013). Les plus vulnérables, en particulier, sont confrontés à de nouveaux choix dans l'allocation de leur budget qui les contraignent à privilégier des calories peu chères mais pauvres nutritionnellement. L'existence d'un (c triple fardeau de la malnutrition), à savoir la concomitance dans un même pays voire au sein d'un même ménage de sous-alimentation, carences nutritionnelles et surconsommation, est aujourd'hui une préoccupation majeure dans les pays en transition.

Le développement des chaînes de valeur, en particulier la transformation alimentaire, offre de nombreuses possibilités en matière d'amélioration nutritionnelle des aliments. L'Afrique de l'Ouest a déjà été le lieu d'expérimentation et mise en place de programmes réussis de fortification des aliments - ou enrichissement en nutriments. Les carences en vitamines et minéraux constituent un véritable problème de santé publique dans le monde et en particulier en Afrique sub-saharienne. En Afrique, la proportion d'enfants de moins de cinq ans affectés par une carence en vitamines $\mathrm{A}^{7}$ est de $44.4 \%$ (OMS, 2009), tandis que

Prendre en compte au sein des systèmes d'information les contraintes des populations vulnérables urbaines. l'anémie, conséquence d'une déficience en fer, touche $67.6 \%$ des enfants de moins de cinq ans et $47.5 \%$ des femmes de 15 à 50 ans $^{8}$ (OMS, 2008). La fortification des huiles en vitamines A et des farines de blé tendre en fer et acide folique est déjà effective dans la plupart des pays ouest-africains. Certains plats transformés,

comme le gari au Bénin, sont enrichis en protéines et/ou vitamine A à partir du soja et/ ou d'huile de palme. La question de la fortification est d'autant plus importante que les procédés de transformation alimentaire peuvent par ailleurs être également source de déperdition en nutriments. Enfin, les activités - notamment de distribution - peuvent donner accès à une gamme plus large de produits et toucher un plus grand nombre de consommateurs. Les acteurs de ces branches d'activités doivent être pleinement représentés dans les plateformes de dialogue sur les questions de sécurité alimentaire.

\section{Développer une capacité d'analyse et de prévention adaptée à la vulnérabilité urbaine}

Des millions d'urbains vivent sous le seuil de pauvreté et souffrent de malnutrition aigüe et/ou chronique. Les dynamiques de vulnérabilité de cette population croissante sont soumises à des caractéristiques, contraintes et ressources spécifiques. Il y a aujourd’hui presque autant d'urbains que de ruraux en Afrique de l'Ouest, et presque autant de personnes en insécurité alimentaire et nutritionnelle en ville qu'en milieu rural. Au Mali, les données des enquêtes à indicateurs multiples (MICS) indiquent que 14 \% des enfants urbains de moins de cinq ans souffrent d'insuffisance pondérale modérée ou sévère, contre $20 \%$ en milieu rural ; $18 \%$ contre $31 \%$ accusent un retard de croissance modéré ou sévère ; $8 \%$ contre $9 \%$ peuvent être considérés comme émaciés de façon modérée ou sévère ; 66 \% contre 74 \% sont touchés par l'anémie (UNICEF et al., 2013). Ces prévalences sont également des marqueurs de l'état nutritionnel de la population totale sous-jacente et soulignent l'ampleur de l'enjeu.

À l'heure actuelle, les dispositifs d'alerte précoce reposent sur une désagrégation géographique des zones d'insécurité alimentaire et nutritionnelle, un zonage, qui ne distingue pas toujours explicitement milieu urbain et milieu rural. Il s'ensuit que les indicateurs de suivi ne sont parfois pas appropriés au milieu urbain. Par exemple, les éléments proposés pour suivre les avoirs des ménages relatifs à leurs moyens d'existence (avoirs productifs agricoles, infrastructures agro-pastorales, eau, alimentation du bétail, aires de pâturage, etc.) ont très peu de sens dans un contexte urbain. La prise en compte

7 Indiquée par un taux de rétinol sérique inférieur au seuil recommandé ( $<0.7 \mu$ mole/L).

8 Pour les femmes non enceintes. La prévalence est de $57.1 \%$ pour les femmes enceintes (OMS, 2008). 
au sein des systèmes d'information de capacités ciblées aux contraintes des populations vulnérables urbaines permet une évaluation plus juste de la situation de la sécurité alimentaire et nutritionnelle, ouvrant le champ à des réponses plus adaptées notamment en cas de crises.

\section{CONCLUSION}

Les transformations des agro-industries, de la commercialisation alimentaire et de l'agriculture sont inextricablement liées et participent de la sécurité alimentaire. La chaîne de valeur reflète bien les réalités de l'économie alimentaire et ses mutations. Un suivi de l'ensemble de la chaîne et une amélioration de son efficacité sont aujourd'hui indispensables pour assurer disponibilité, accessibilité, qualité et stabilité des approvisionnements alimentaires. Cette vision multisectorielle permet d'élaborer des politiques de sécurité alimentaire et nutritionnelle plus en phase avec les mutations de l'économie alimentaire, et d'améliorer les mécanismes de résilience.

La nature et l'étendue de la demande alimentaire offrent des opportunités de diversification et de création de valeur ajoutée. Pour que les exploitations agricoles familiales, qui sont et demeureront majoritaires, puissent en profiter, il importe de les intégrer dans les chaînes de valeur. C'est le moyen d'ajuster leurs offres aux nouvelles exigences de la demande et leur permettre d'accéder à ces marchés. Les activités aval de l'agriculture représentent aussi un important vivier de création d'emplois - en particulier féminins - et de sources de revenu. Pour générer les emplois, les revenus et l'alimentation nécessaire à une population croissante, il faut accompagner ces changements de l'économie alimentaire de manière aussi profonde que l'agriculture. 


\section{RÉFÉRENCES}

BAD (2014), L'industrie agroalimentaire en Afrique de l'Ouest, Observatoire de l'Afrique de l’Ouest, numéro 4, janvier 2014, Banque africaine de développement (BAD), Abidjan, Côte d'Ivoire.

Banque mondiale (2015), (( Global consumption database ) (base de données), http://datatopics.worldbank.org/consumption/ (consulté le 20 avril 2016).

Bricas, N. et C. Tchamda (2015), Les profondes mutations des consommations alimentaires en Afrique, F. Mouton (éd.), Question de développement, Agence française de développement (AFD), Paris.

DPEE Senegal (2015), « Direction de la Prévision et des Études Économiques - Ministère de l'Économie et des Finances du Sénégal ) (Base de données) http://www.dpee. sn/-Structure-du-PIB,123-.html?lang=fr (consulté le 7 septembre 2015).

FAO (2010), « Agricultural value chain development. Threat or opportunity for women's employment? s), Gender and rural employment policy brief 4, FAO, Rome.

FAO (1997), (c Les industries agroalimentaires et le développement économique ), in La situation mondiale de l'alimentation et de l'agriculture, FAO, Rome.

FAO, FIDA et PAM (2013), L'état de l'insécurité alimentaire dans le monde 2013 : les multiples dimensions de la sécurité alimentaire, FAO, Rome.

FAOSTAT (2015), « Food and Agriculture Organization of the United Nations - Statistics Division ») (base de données). http://faostat3.fao.org/home/E (consulté le 7 septembre 2015).

Henriksen, L.F., L. Riisgaard, S. Ponte, F. Hartwich et P. Kormawa (2010), « Agro-food value chain interventions in Asia: A review and analysis of case studies m, document de travail, United Nations Industrial Development Organization (UNIDO), Vienne.

Henson, S. et J. Cranfield (2009), « Building the political case for agroindustries and agribusiness in developing countries s), in Agro-industries for development, pp. 10-46.

Hollinger, F. et J. Staatz (eds.) (2015), Agricultural growth in West Africa: Market and policy drivers. Food and Agricultural Organization of the United Nations (FAO), Rome.

ICP 2011 (2015), “ International Comparison Program 2011 - Banque mondiale ») (Base de données) http://siteresources.worldbank.org/ICPEXT/Resources/ICP_2011.html (consulté le 7 septembre 2015).

INSTAT (2013), ( Enquête par grappes à indicateurs multiples 2010 Mali ), Institut national de la statistique (INSTAT), Cellule de planification et de statistique du secteur santé, développement social et la promotion de la famille (CPS/SSDSPF), Rapport final, Bamako, Mali.

Moriconi-Ebrard, F., D. Harre et P. Heinrigs (2016), L'urbanisation des pays de l'Afrique de l'Ouest 1950-2010 : Africapolis I, mise à jour 2015, Cahiers de l'Afrique de l'Ouest, Éditions OCDE, Paris, http://dx.doi.org/10.1787/9789264252257-fr.

OCDE (2016), GVC Participation in the Agriculture and Food Sectors, Éditions OCDE, Paris (à paraître). 
OCDE (2015), « Africapolis Liste et Population des agglomérations ouest-africaines 1950-2010 ), Statistiques Afrique de l'Ouest (base de données), http://stats.oecd.org/ (consulté le 20 avril 2016).

OCDE (2013), Peuplement, marché et sécurité alimentaire, Cahiers de l'Afrique de l'Ouest, Éditions OCDE, Paris, http://dx.doi.org/10.1787/9789264187412-fr.

OMS (2009), Global prevalence of vitamin A deficiency in populations at risk 1995-2005, WHO Global database on vitamin A deficiency, OMS, Genève.

OMS (2008), Worldwide prevalence of anaemia 1993-2005: WHO Global database on anaemia, Organisation mondiale de la santé (OMS), Genève.

Paillard, S., S. Treyer et B. Dorin (2010), Agrimonde. Scénarios et défis pour nourrir le monde en 2050, Matière à débattre et à décider, Éditions Quae, Paris.

Réseau pour l'emploi des jeunes et Fondation internationale de la jeunesse (2009), Private sector demand for youth labour in Ghana and Senegal: Ghana and Senegal study findings, Banque mondiale, Washington DC et BIT, Genève.

Tschirley, D., T. Reardon, M. Dolislager et J. Snyder (2014), « The rise of a middle class in East and Southern Africa: Implications for food system transformation ), Journal of International Development, vol. 27, no 5, pp. 628-646.

UNSD (2015), “United Nations Statistics Division - National Accounts Statistics: Main Aggregates and Detailed Tables" (base de données) http://unstats.un.org/unsd/ nationalaccount/madt.asp?SB=1\&\#SBG (consulté le 7 septembre 2015). 